\title{
Radiologisch-interventionelle Drainage bei abdomineller Sepsis
}

\author{
Antonia Wiggermann Peter Hunold \\ Klinik für Radiologie und Nuklearmedizin, Universitätsklinikum Schleswig-Holstein, Campus Lübeck, Deutschland
}

\author{
Schlüsselwörter \\ Septische Dissemination - Abszess . \\ CT-gesteuerte Intervention · Drainage · \\ Interventioneller Eingriff
}

\section{Zusammenfassung}

Hintergrund: Durch sekundäre Infektion pankreatischer Nekrosen und Pseudozysten, putride enterische Infektionen und infolge abdominalchirurgischer Eingriffe kann es zur Ausbildung intraabdomineller Abszesse kommen. Diese stellen Foci septischer Dissemination dar. Die anatomische Lage sowie Ausbreitung lässt sich mittels Computertomographie (CT) exakt bestimmen. Einfache Abszesse sind eindeutig abgrenzbar und treten singulär auf. Komplexe Abszessformationen sind häufig multifokal lokalisiert. Bei Abszessformationen $>50 \mathrm{~mm}$ ist die Indikation zur radiologisch-interventionellen oder chirurgischen Sanierung zu stellen. Methode: In Abhängigkeit von der topographischen Lage des Abszesses erfolgt der Zugang von ventral, perineal, vaginal oder transgluteal. Abszesspunktion und Drainageanlage werden in Direktpunktions-, Tandem-Trokar- oder Seldinger-Technik vorgenommen. Die Punktion wird unter bildgebender Kontrolle mit Fluoroskopie oder Sukzessivsequenzen durchgeführt. Ergebnisse: Die CT-gesteuerte Anlage einer perkutanen Abszessdrainage (PAD) ist im Fall unkomplizierter und gut zugänglicher intraabdomineller Abszesse die Therapie der Wahl. Eine mögliche Verletzung wichtiger Strukturen auf dem Punktionsweg, wie z.B. von Darm, Milz oder großen Gefäßen, stellt jedoch eine Kontraindikation der PAD dar. Schlussfolgerung: Die PAD ist ein sicheres radiologisch-interventionelles Verfahren. Bei geringerer Krankenhausverweildauer und punktionsbedingter Morbidität ist ihr therapeutischer Erfolg mit jenem der chirurgischen Fokussanierung gleichzusetzen.
Keywords

Septic dissemination - Abscess .

CT-guided percutaneous drainage - Drainage .

Interventional procedure

\section{Summary}

\section{CT-Guided Percutaneous Drainage of Intraabdominal} Abscesses

Background: Abdominal abscesses are caused by putrid infections of the intestine and the abdominal cave as well as by pancreatic pseudocysts. They are foci of septic dissemination. Anatomic location and intraabdominal spread can be exactly determined by computed tomography (CT) imaging. Here, simple abscesses are clearly distinguishable. Complex abscesses remain multilocular. Radiological or surgical drainage is indicated in abscesses $>50 \mathrm{~mm}$. Method: Depending on the anatomic site, draining of the abscess can be performed from ventral, perineal, vaginal, or transgluteal. Different methods such as direct puncture, tandem-trocar and Seldinger technique are described. During puncture CT imaging is performed by fluoroscopy or successive CT images. Results: CT-guided percutaneous drainage (PAD) is the recommended first-line therapy of uncomplicated and easily accessible abdominal abscesses. The possible injury of vital structures, such as intestine, spleen, or large blood vessels, means a contraindication. Conclusion: PAD is a safe interventional technique. Its therapeutic success is comparable to surgical intervention, with less puncture-related morbidity and a shorter hospital stay.

\begin{tabular}{ll}
\hline KARGER & @ 2013 S. Karger GmbH, Freiburg \\
Fax +49 761 452 07 14 & 1662-6664/13/0291-0014\$38.00/0 \\
$\begin{array}{l}\text { Information@Karger.com } \\
\text { www.karger.com }\end{array}$ & $\begin{array}{l}\text { Accessible online at: } \\
\text { www.karger.com/vim }\end{array}$
\end{tabular}




\section{Einleitung}

Der Begriff Sepsis beschreibt einen klinischen Symptomenkomplex als systemische Reaktion des Organismus auf die hämatogene Aussaat mikrobieller Antigene. Intraabdominelle Abszesse stellen septische Foci dar. Häufige Ursachen in der Viszeralchirurgie sind Hohlorganperforationen, Divertikulitiden, Appendizitis, chronisch-entzündliche Darmerkrankungen (CED) und die nekrotisierende Pankreatitis. Auch eine Anastomoseninsuffizienz kann die Ursache eines Abszesses sein. Vor der Einführung moderner Antibiotika stellte die offen chirurgische Sanierung die einzige kausale Therapieoption dar $[1,2]$ und war mit einer hohen Mortalität behaftet. In Abhängigkeit von der anatomischen Lokalisation, Größe und Beschaffenheit variiert diese zwischen $28 \%$ und bis zu $100 \%$ bei nicht sanierbarer Sepsisquelle [3-5]. Die Einführung der Computertomographie (CT) und die daraus resultierende Entwicklung CT-gesteuerter perkutaner Punktionstechniken eröffneten neue diagnostische und therapeutische Optionen bei abdominellen Abszessen (perkutane Abszessdrainage, PAD). Die CT-gesteuerte perkutane Drainage stellt heutzutage die Standardtherapie abdomineller Abszesse dar [6].

\section{Perkutane Abszessdrainage: Erfolgsfaktoren und therapeutischer Nutzen}

Bei zu hoher Viskosität des Abszessinhalts oder direkter topographischer Nähe zu wichtigen Strukturen, wie z.B. Darm, Milz und großen Gefäßen, stellt die PAD aufgrund der mangelnden Erfolgsaussichten oder des bestehenden Komplikationsrisikos keine Therapieoption dar. Die transpleurale Punktion subphrenischer Abszesse birgt das Risiko der Ausbildung eines Pleuraempyems. Die chirurgische Fokussanierung mit Debridement und Drainageanlage ist hier weiterhin die Therapie der Wahl.

Hinsichtlich Morbidität und Mortalität bestehen bei gut zugänglichen Abszessen keine signifikanten Unterschiede zwischen chirurgischer Abszessdrainage (CAD) und PAD. In einer von Olak et al. [7] bei 54 Patienten durchgeführten FallKontroll-Studie betrug die Mortalität nach PAD 11\% versus $7,4 \%$ nach CAD. Zudem beobachteten die Autoren eine Morbidität von 29\% (PAD) versus 40\% (CAD). Der therapeutische Erfolg betrug 70\% (PAD) versus $85 \%$ (CAD). Hemming et al. [8] berichten in der von ihnen an 84 Patienten durchgeführten Fall-Kontroll-Studie deutlich geringere Unterschiede im Vergleich der Methoden: Sie beschreiben eine Mortalität von 12\% (PAD) versus 14\% (CAD) und eine Morbidität von $29 \%$ (PAD) versus $26 \%$ (CAD). Ihr Therapieerfolg liegt bei 93\% (PAD) bzw. 96\% (CAD). Auch die Krankenhausverweildauer war in beiden Kollektiven ähnlich. Geringere Erfolgsraten werden bei der PAD von CED-assoziierten Abszessen berichtet. In diesem Fall ist in 27-61\% von einem Therapieerfolg auszugehen [9-11]. Die PAD in der Be- handlung der chronisch-rezidivierenden Abszesse ist hier deutlich der Chirurgie überlegen [12]. Eine kalkulierte antibiotische, bei Risikopatienten gegebenenfalls auch antimykotische Therapie ist hier unbedingt frühzeitig nach der Abnahme von Blutkulturen einzuleiten und stellt einen primären Bestandteil der Therapie dar [13].

Hinsichtlich eines rein konservativen Vorgehens (Antibiotikatherapie) scheint die Größenausdehnung der Abszessformation ein entscheidender prädiktiver Faktor zu sein. So berichten Bamberger et al. [14], dass bei abdominellen Abszessen $<50 \mathrm{~mm}$ häufig bereits die alleinige antibiotische Therapie ausreichend sein soll. Abszesse $>50 \mathrm{~mm}$ bedürfen hingegen einer zusätzlichen radiologischen (PAD) oder chirurgischen (CAD) Intervention.

\section{Präinterventionelle Diagnostik der perkutanen Abszessdrainage}

Dank ihrer hohen Auflösung und der damit verbundenen exakten Darstellung der anatomischen Verhältnisse stellt die CT mit oraler und intravenöser Kontrastierung heute die Methode der Wahl in der Diagnostik von abdominellen Abszessen dar. Somit lassen sich perkutane Zugangswege unter Umgehung angrenzender anatomischer Strukturen zuverlässig und sicher planen. Abszessformationen sind oft unscharf begrenzt und zeigen native Dichtewerte von 0-25 HoundsfieldEinheiten. Folglich kann mittels CT nicht sicher zwischen sterilen und infektiösen Flüssigkeitsansammlungen differenziert werden $[15,16]$. In diesem Fall kann additiv die Sonographie zur weiteren Differentialdiagnostik hinzugezogen werden: Sterile, abgekapselte Flüssigkeitsverhalte stellen sich hier echoarm dar. Abszessformationen dagegen imponieren als unscharf begrenzte, von echoreichen Binnenechos durchsetzte Raumforderung. Zur sicheren Unterscheidung zwischen abszesssuspekter, paraenterischer Raumforderung und dem Darmlumen ist eine intraluminale Kontrastmittelapplikation indiziert.

\section{Indikationen und Kontraindikationen der perkutanen Abszessdrainage}

Besteht der klinisch-radiologische Verdacht auf das Vorliegen eines Abszesses im Abdomen, ist prinzipiell die Indikation zur diagnostisch-therapeutischen PAD zu stellen. Mittels Drainage des septischen Focus ist eine Materialgewinnung für die mikrobiologische und laborchemische Diagnostik möglich, die dann eine zielgerichtete antibiotische Therapie erlaubt und aufgrund des Erregerspektrums oft Hinweise auf die Sepsisquelle bietet. Bei Nachweis von Lipase oder hohem Kreatinin im Sekret ergeben sich gegebenenfalls Hinweise auf eine gastrointestinale Nahtinsuffizienz, eine Pankreasfistel und eine Leckage im Urogenitaltrakt als Abszessursache. 
Abb. 1. Zustand nach Chemotherapie bei Ösophaguskarzinom mit Leberabszess. a Planungs-CT ohne Kontrastmittel.

b Markierung der avisierten Hautinsertionsstelle lateral mit einer Metallkanüle. c Einführung der
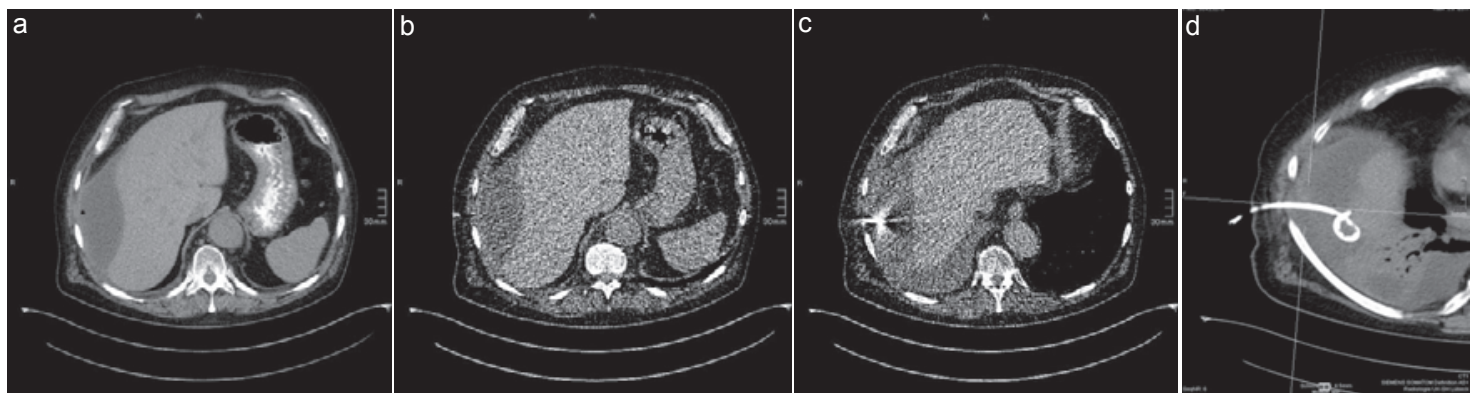

Koaxialnadel. d Konfigurierter Pigtailkatheter im Verhalt nach Entfernen des Trokars.

Die Evakuierung des Abszessinhalts mittels PAD stellt neben der systemischen Antibiotikatherapie die wichtigste kausale Therapie dar. Die topographische Ausdehnung der Abszessformation stellt die Entscheidungsgrundlage für die Anlage einer PAD oder einer singulären, CT-gesteuerten Punktion dar [17]. Relative Kontraindikationen der PADAnlage stellen eine hämodynamisch instabile Kreislaufsituation, Blutgerinnungsstörungen, Noncompliance und andere schwerwiegende Beeinträchtigungen des klinischen Zustands des Patienten dar [15, 16, 18-24]. Ist kein sicherer Zugangsweg ohne das Verletzungsrisiko des Darms, großer Blutgefäße oder anderer lebenswichtiger Organe und Strukturen gegeben, so ist die Anlage einer PAD kontraindiziert. In diesem Fall ist eventuell lediglich eine diagnostische Punktion möglich. Vorgeschaltete Organstrukturen werden hierbei mittels einer 22-Gauge-Chiba-Nadel minimaltraumatisch passiert [19, 25-27]. Bei unkooperativen, motorisch unruhigen Patienten ist in der interdisziplinären Absprache zwischen Chirurg, interventionellem Radiologen und Anästhesist abzuwägen, ob die Intervention in Narkose erfolgen kann oder ob eine chirurgische Intervention indiziert ist.

\section{Technik der CT-gesteuerten Drainagenanlage}

Präinterventionell ist vor PAD-Anlage die Bestimmung der plasmatischen und der thrombozytären Gerinnungssituation zur Abschätzung des Risikos von Blutungskomplikationen vorzunehmen. Mit dem behandelnden Arzt des Patienten ist zu besprechen, ob die Unterbrechung einer etwaigen medikamentösen Antikoagulation möglich ist oder ob diese Medikation eine Kontraindikation für das radiologisch-interventionelle Verfahren darstellt.

Je nach Lokalisation der Abszessformation und dem gewählten Zugangsweg wird der Patient in Interventionsposition gelagert. Zur exakten Evaluation der Abszesslokalisation erfolgt in Interventionsposition unmittelbar vor Durchführung der Punktion ein Planungs-CT. Nach der Markierung der Punktionsstelle wird diese großflächig desinfiziert und steril abgedeckt. 10-20 ml eines Lokalanästhetikums werden bedarfsadaptiert subkutan und intramuskulär injiziert. Der
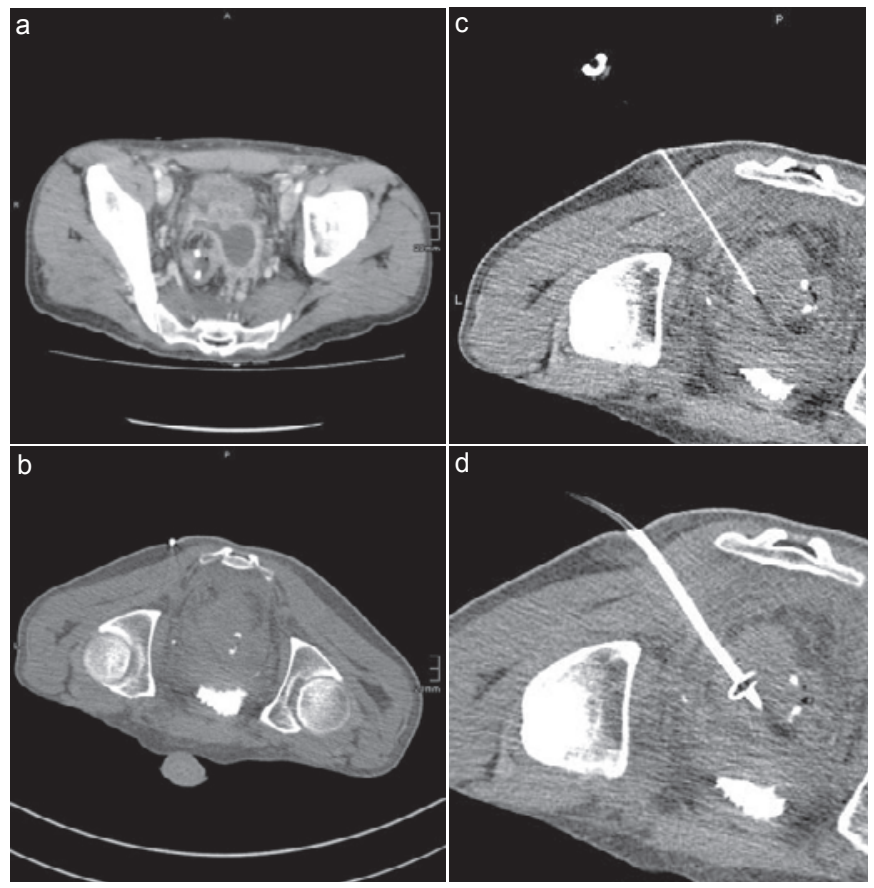

Abb. 2. Anastomoseninsuffizienz mit Abszess bei Zustand nach Ileoileostomie: Drainage mit einem Malecot-Körbchenkatheter. a Diagnostisches CT mit Kontrastmittel in Rückenlage. b Interventions-CT in Bauchlage ohne Kontrastmittel: Markierung der geplanten Punktionsstelle auf der Haut rechts paramedian mit einer metallischen Kanüle. c Einführung der Koaxialkanüle zur Einbringung des Seldinger-Drahts. d Abschlusskontrolle: Malecot-Körbchenkatheter im Abszess.

Trokar bzw. die Nadel wird CT-gesteuert eingebracht. Dies erfolgt entweder durch Fluoroskopie (höhere Strahlungsdosen) oder mittels Sukzessivsequenzen in etwa $5 \mathrm{~mm}$ Schichtdicke. Nach Abschluss der Intervention wird zur Erfolgskontrolle und zum Ausschluss von Komplikationen eine Kontroll-CT durchgeführt (Abb. 1,2).

\section{Abdominelle Zugangswege der perkutanen Abszessdrainage}

Die PAD-Anlage kann über unterschiedliche Zugangswege nach intraabdominell erfolgen: 


\section{Anteriorer transabdomineller Zugangsweg}

Hierbei handelt es sich um den technisch einfachsten und somit meistgenutzten Zugangsweg nach intraabdominell. Dennoch ist dieser nicht in jedem Fall von Vorteil. Insbesondere bei tief intraperitonealen oder retroperitonealen Abszessformationen kann die Punktionsstrecke durch vorgeschaltete Bauchorgane oder Darmschlingen verlegt sein. Zudem besteht hier ein Verletzungsrisiko der epigastrischen Gefäße [28-30].

\section{Lateraler transabdomineller Zugangsweg}

Hier erfolgt die Punktion der Bauchwand von lateral. Die radiologische Darstellung der Iliakalgefäße ist hierbei zwingend erforderlich, da diese entlang der lateralen, vorderen Bauchwand verlaufen (Abb. 1).

\section{Transglutealer Zugangsweg}

Der Zugang zum kleinen Becken erfolgt über das Foramen ischiadicum unter Passage der glutealen Muskulatur (Abb. 2). Dieser Punktionsweg ist insbesondere bei tief pelvinen Abszessformationen, hervorgerufen beispielsweise durch Divertikulitiden, indiziert, da sich diese oft der Punktion und Drainage von anterior entziehen. Der Punktionsweg ist optimalerweise durch das Ligamentum sacrospinosum und den Musculus piriformis hindurch zu wählen, um das Verletzungsrisiko des Plexus ischiadicus zu minimieren. Eine postpunktionelle Schmerzsymptomatik stellt hier eine häufige Begleitkomplikation dar [31].

\section{Transperinealer Zugang}

Der Zugang von transperineal ist ebenfalls bei tief- bzw. subpelvinen Abszessformationen, die insbesondere präsakral oder pararektal lokalisiert sind, indiziert. Diese können nach abdominoperinealer Rektumresektion und Bestrahlungstherapie von Rektumkarzionomen auftreten. Dieser Zugangsweg ist technisch anspruchsvoll. Entsprechend sollte hier die Punktion unter Real-time-Bedingungen, z.B. mittels sonographischer Kontrolle, erfolgen [32-34].

\section{Transvaginaler Zugang}

Bei ventralen, tief pelvinen Abszessformationen infolge von Adnexitiden oder auch Divertikulitiden kann alternativ eine Punktion von transvaginal erfolgen. Auch hier ist eine sonographische Real-time-Darstellung des Punktionsvorgangs sinnvoll [35-40].

\section{Punktionstechniken}

In der Literatur wird zwischen drei unterschiedlichen technischen Vorgehensweisen bei Anlage einer PAD unterschieden:

- direkte PAD-Anlage,

- Tandempunktion,

- Seldinger-Technik [15-23, 41-49].
Die Abszessdrainage in Direktpunktionstechnik erfolgt über einen sogenannten Introducer. Der Drainagekatheter liegt diesem System von außen an. Innen verlaufen eine sogenannte Versteifungskanüle und der Punktionstrokar. Das Gewebe und die Abszessmembran werden mit dem Punktionstrokar penetriert. Die Versteifungskanüle liegt zwischen dem Drainagekatheter und dem Punktionstrokar. Hierdurch werden die auf das umliegende Gewebe einwirkenden Reibungskräfte reduziert.

Eine Sonderform der Direktpunktion ist die sogenannte Tandem-Trokar-Technik. Die Abszesspunktion erfolgt primär mit einer 18-, 20- oder 22-Gauge-Hohlnadel ohne die direkte Drainageanlage. Für die weitere mikrobiologische Diagnostik wird über diese zunächst Abszessinhalt aspiriert. Die Drainageanlage erfolgt in einem zweiten Schritt entlang des primären Punktionskanals.

Die Seldinger-Technik beschreibt ein Vorgehen, bei dem zunächst ein Führungsdraht über eine Hohlnadel in die Abszessformation positioniert wird. Über diesen wird anschlieBend das Drainagesystem angelegt.

Aufgrund der komplexeren Vorgehensweise kommen Tandem-Trokar- und Seldinger-Technik bei großen, gut zugänglichen Abszessen seltener zum Einsatz. Da die primäre Sondierung der Abszessformation in diesem Fall jedoch über dünnere Nadeln erfolgt, eignen sich diese Techniken bei komplizierteren Zugangswegen, erhöhtem Verletzungsrisiko vorgelagerter anatomischer Strukturen und kleineren Abszessen.

\section{Diagnostische Aspiration}

Computertomographisch lassen sich keine Aussagen über den Infektionserreger einer abszesssuspekten abdominellen Raumforderung ableiten $[15,16]$. Die mikrobiologische und/ oder laborchemische Diagnostik ist aufgrund dieser Tatsache mit dem nach primärer Punktion gewonnenen Aspirat vorzunehmen. $\mathrm{Zu}$ beachten gilt hier jedoch, dass ein negativer Bakteriennachweis in der Gramfärbung einen Abszess bei antibiotisch vorbehandelten Patienten nicht ausschließt. Leukozyten lassen sich bei immunsupprimierten oder chemotherapeutisch vorbehandelten Patienten ebenso oft nicht nachweisen. Ist das gewonnene Aspirat klarflüssig, sollten in Abhängigkeit von der anatomischen Lokalisation die Kreatininkonzentration und die Amylaseaktivität bestimmt werden. Auf diese Weise lassen sich Urinome und Pankreasfisteln differentialdiagnostisch unterscheiden.

\section{Kathetertypen}

In der klinischen Praxis kommen insbesondere zwei Typen von Kathetersystemen zum Einsatz: der sogenannte Pigtailkatheter und der Malecot-Körbchenkatheter (Abb. 3). 
Abb. 3. Kathetertypen.

a Pigtailkatheter.

b Malecot-Körbchen-

katheter.
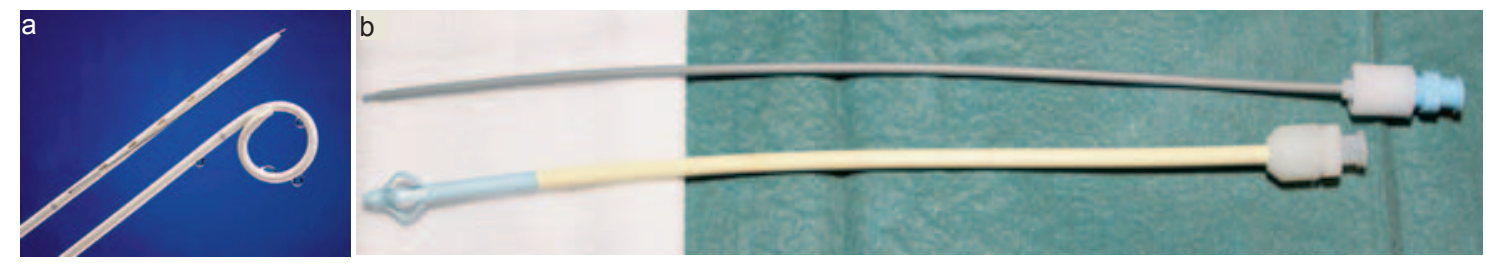

Der Pigtailkatheter besitzt eine am distalen Katheterende lokalisierte Schlaufe. Diese dient der Fixierung des Katheters in seinem Zielgebiet. Die distale Windung wird bei Einbringen des Katheters durch den Stabilisierungseffekt der Versteifungskanüle aufgehoben. Somit wird ein Aufrollen des Seldinger-Drahtes bei Vorschub des Katheters unterbunden. Erst nach Punktion des Abszesses und Rückzug der Versteifungskanüle entfaltet der Katheter seine gewundene Form.

Der Malecot-Körbchenkatheter ist gerade. Am distalen Katheterende befindet sich eine Draht- oder Kunststoffspindel. Diese verhindert, ähnlich der Windung des Pigtailkatheters, dessen Dislokation. Die Kunststoffspindel wird bei Einbringen des Katheters mittels eines sogenannten Streckersystems gestreckt und liegt somit plan in der Ebene der äußeren Katheterwand. Nach Entfernung des Streckersytems entfaltet sich die Spindel.

\section{Drainage von Organabszessen}

Intraabdominelle Abszesse können in unterschiedlichen Organen und anatomischen Regionen auftreten. Nachfolgend wird ein Überblick über die häufigsten abdominellen Abszessmanifestationen und ihr therapeutisches Management gegeben:

\section{Leberabszess}

Der Entstehung von Leberabszessen liegt meist eine kompromittierte mukosale Immunabwehr des Gastrointestinaltrakts zugrunde. Weitere Ursachen stellen abdominalchirurgische Eingriffe bzw. endoskopische Interventionen und Gallenwegserkrankungen dar [50,51]. Leberabszesse können isoliert oder multipel auftreten [52]. Isolierte Leberabszessse treten gehäuft im rechten Leberlappen auf. Multiplen Leberabszessen liegt oftmals eine Infektion mit Escheria coli zugrunde. Diese sind häufig in beiden Leberlappen lokalisiert. Unbehandelte Leberabszesse haben eine infauste Prognose. Eine systemische Antibiotikatherapie und eine radiologisch-interventionelle oder chirurgische Fokussanierung sind hier umgehend einzuleiten. Insbesondere bei multiplen Leberabszessen ist die Indikation zur PAD eng und frühzeitig zu stellen. Auf diese Weise lassen sich Heilungsraten von bis zu 98\% erzielen [53].

\section{Pankreasabszess}

Infolge einer akuten nekrotisierenden Pankreatitis kommt es zu einer phlegmonösen Imbibierung des Pankreas. Lassen sich freie, peripankreatische Flüssigkeitsformationen und eine lokoregionär unterbrochene Kontrastmittelaufnahme des Pankreas im CT nachweisen, so ist radiologisch von einer akuten nekrotisierenden Pankreatitis auszugehen. Während des weiteren Verlaufs kommt es nicht selten innerhalb von 1-4 Wochen zur Ausbildung pankreatischer Pseudozysten [54]. Durch die sekundäre Infektion pankreatischer Nekrosen oder Pseudozysten kann sich ein Pankreasabszess ausbilden. In diesem Fall ist eine umgehende Drainage indiziert [47, 55-57]. Aufgrund der exakten topographischen Darstellung abszesssuspekter pankreatischer Raumforderungen stellt hier die CT-gesteuerte Punktion die Therapie der Wahl dar. Diese kann entweder über einen transperitonealen oder transretroperitonealen Zugangsweg erfolgen. Nach einmaliger Punktion sind pankreatische Abszesse in bis zu 70\% rekurrent. Nach Anlage einer PAD kommt es deutlich seltener zum Rezidiv [57, 58]. Der technische Erfolg dieser Maßnahme liegt zwischen 70 und 100\% [47, 55-57, 59].

\section{Enterischer Abszess}

Appendizitis, Divertikulitis und CED stellen die häufigsten Ursachen für die Entwicklung paraenterischer Abszessformationen dar. Parakolische Abszesse infolge einer Divertikulitis oder Appendizitis lassen sich erfolgreich mittels PAD drainieren. Der Patient kann somit für eine spätere definitive chirurgische Resektion vorbereitet werden [60]. Paraenterische Abszesse infolge von CED entstehen aufgrund einer durch die Erkrankung eingeschränkten mukosalen Immunität. Die PAD ist hier in bis zu $67 \%$ der Fälle erfolgreich und der definitiven Chirurgie überlegen [9-11]. Postoperative Abszesse, z.B. aufgrund einer Anastomoseninsuffizienz, gehen mit einer Mortalität von bis zu 30\% einher [61]. Nach Diagnose eines postoperativen septischen Focus kann die PAD zur Konditionierung für eine spätere definitive chirurgische Revision erfolgen. In Einzelfällen stellt diese bereits eine endgültige Ursachenbeseitigung und Therapie dar [62].

\section{Milzabszess}

Die hämatogene Dissemination von Bakterien und deren Absiedlung kann zur Ausbildung splenischer Abszesse führen. In der CT imponieren sie als hypodense Läsionen mit peripherer Kontrastmittelanreicherung. Mit einer Therapieerfolgsquote von $75-100 \%$ stellt die PAD eine wichtige interventionelle Therapieoption in der Behandlung splenischer Abszesse dar [63-66]. Die Patienten sollten nach Punktion und Drainage engmaschig überwacht werden, um etwaige Blutungskomplikationen sofort zu erkennen und zu behandeln. 


\section{Schlussfolgerung}

Die PAD zeigt bei intraabdominellen Abszessen einen therapeutischen Erfolg von bis zu 93\%. Der technische Erfolg der radiologischen Intervention korreliert nicht immer mit der Sanierung der Sepsis. Genaue Daten aus großen Studien fehlen hierzu. Für komplexe, mit fistulierenden Prozessen assoziierte Abszessformationen ist die Datenlage auch für den technischen Erfolg nicht eindeutig. Unterschiedliche Autoren berichten von Erfolgsquoten der perkutanen Therapie, die sich zwischen 45 und $88 \%$ bewegen [12, 18, 67-69]. Gemäß den Leitlinien des American College of Radiology (ACR) ist die Anlage einer PAD als primäre therapeutische Option bei folgenden Erkrankungsbildern zu empfehlen:

- Einfache Abszesse mit sicherem Punktionsweg

- Komplexe Abszesse mit sicherem Punktionsweg

- Singuläre oder multiple Leberabszesse

- Infizierte pankreatische Pseudozysten
Multilokuläre Abszessformationen, eine hohe Viskosität des Abszessinhalts, Kachexie, Patientenalter und ein hoher APACHE-II-Score (Acute Physiology Score + Age Points + Chronic Health Points) stellen Prädiktoren eines negativen Therapieerfolgs dar [52]. Mit einer direkten, punktionsbedingten Mortalität von $0,7 \%$ und einer Komplikationsrate von insgesamt $5-11 \%$ stellt die PAD eine sichere therapeutische Alternative zum chirurgischen Vorgehen dar [15, 16, 18, 20-22, 41, 70]. Eine interdisziplinäre Therapieplanung im Konsens mit Chirurgen und Internisten kann die periinterventionelle Therapieplanung verbessern und bestehende Risiken minimieren.

\section{Disclosure Statement}

Der korrespondierende Autor gibt an, dass kein Interessenkonflikt besteht.

\section{Literatur}

1 Altemeier WA, Culbertson WR, Fullen WD, Shook CD: Intra-abdominal abscesses. Am J Surg 1973;125:70-79.

2 Malangoni MA, Shumate CR, Thomas HA, Richardson JD: Factors influencing the treatment of intra-abdominal abscesses. Am J Surg 1990;159: 167-171.

$>3$ Levison MA: Percutaneous versus open operative drainage of intra-abdominal abscesses. Infect Dis Clin North Am 1992;6:525-544.

4 Montgomery RS, Wilson SE: Intraabdominal abscesses: image-guided diagnosis and therapy. Clin Infect Dis 1996;23:28-36.

$\checkmark 5$ Branum GD, Tyson GS, Branum MA, Meyers WC: Hepatic abscess. Changes in aetiology, diagnosis, and management. Ann Surg 1990;212:655-662.

6 Bakal CW, Sacks D, Burke DR, Cardella JF, Chopra PS, Dawson SL, Drooz AT, Freeman N, Meranze SG, Van Moore A Jr, Palestrant AM, Roberts AC, Spies JB, Stein EJ, Towbin R: Society of Interventional Radiology Standards of Practice Committee. J Vasc Interv Radiol 2003;14:S223-225.

7 Olak J, Christou NV, Stein LA, Casola G, Meakins JL: Operative vs percutaneous drainage of intraabdominal abscesses: comparison of morbidity and mortality. Arch Surg 1986;121:141-146.

$\checkmark$ Hemming A, Davis NL, Robins RE: Surgical versus percutaneous drainage of intra-abdominal abscesses. Am J Surg 1991;161:593-595.

9 Garcia JC, Persky SE, Bonis PA, Topazian M: Abscesses in Crohn's disease: outcome of medical versus surgical treatment. J Clin Gastroenterol 2001; 32:409-412.

10 Ayuk P, Williams N, Scott NA, Nicholson DA, Irving $\mathrm{MH}$ : Management of intra-abdominal and pelvic abscess in Crohn's disease. Ann R Coll Surg Engl 1996;78:5-10.

11 Jawhari A, Kamm MA, Ong C, Forbes A, Bartram CI, Hawley PR: Intra-abdominal and pelvic abscess in Crohn's disease: results of noninvasive and surgical management. Br J Surg 1998;85:367-371.
12 Schuster MR, Crummy AB, Wojtowycz MM, McDermott JC: Abdominal abscess associated with enteric fistulas: percutaneous management. J Vasc Interv Radiol 1992;3:359-363.

13 Lee H, Kim YH, Kim JH, Chang DK, Son HJ, Rhee PL, Kim JJ, Paik SW, Rhee JC: Nonsurgical treatment of abdominal or pelvic abscess in consecutive patients with Crohn's disease. Dig Liver Dis 2006:38:659-664.

14 Bamberger DM: Outcome of medical treatment of bacterial abscesses without therapeutic drainage: a review of cases reported in the literature. Clin Infect Dis 1996;23:604-607.

15 Gerzof SG, Robbins AH, Johnson WC, Burkette DH, Nasbeth DC: Percutaneous catheter drainage of abdominal abscesses: a five-year experience. N Engl J Med 1981;305:653-657.

16 Kumar RR, Kim JT, Haukoos JS, Macias LH, Dixon MR, Stamos MJ, Konyalian VR: Factors affecting the successful management of intra-abdominal abscesses with antibiotics and the need for percutaneous drainage. Dis Colon Rectum 2005; 49:183-189.

17 Casola G, vanSonnenberg E, Neff CC, Saba RM, Withers C, Emarine CW: Abscesses in Crohn disease: percutaneous drainage. Radiology 1987;163: 19-22.

18 vanSonnenberg E, Ferrucci JT Jr, Mueller PR, Wittenberg J, Simeone JF: Percutaneous drainage of abscesses and fluid collections: technique, results and applications. Radiology 1982;142:1-10.

19 Lang EK: Renal, perirenal, and pararenal abscesses: percutaneous drainage. Radiology 1990;174: 109-113.

20 Mueller PR, vanSonnenberg E, Ferrucci JT Jr: Percutaneous drainage of 250 abdominal abscesses and fluid collections. Part II. Current procedural concepts. Radiology 1984;151:343-347.

21 vanSonnenberg E, D’Agostino HB, Casola G, Halasz NA, Sanchez RB, Goodacre BW: Percutaneous abscess drainage: current concepts. Radiology 1991;181:617-626.
2 Mueller PR, vanSonnenberg E: Interventional radiology in the chest and abdomen. N Engl J Med 1990;322:1364-1374.

23 Lambiase RE, Deyoe L, Cronan JJ, Dorfman GS: Percutaneous drainage of 335 consecutive abscesses: results of primary drainage with 1-year followup. Radiology 1992;184:167-179.

24 vanSonnenberg E, Wing VW, Casola G, Coons HG, Nakamoto SK, Mueller PR, Ferrucci JT Jr, Halasz NA, Simeone JF: Temporizing effect of percutaneous drainage of complicated abscesses in critically ill patients. AJR Am J Roentgenol 1984;142: 821-826.

25 Ho CS, Taylor B: Percutaneous transgastric drainage of pancreatic pseudocyst. AJR Am J Roentgenol 1984;143:623-625.

26 Mueller PR, Ferrucci JT Jr, Butch RJ, Simeone JF, Wittenberg J: Inadvertent percutaneous catheter gastroenterostomy during abscess drainage: significance and management. AJR Am J Roentgenol 1985;145:387-391.

27 Mueller PR, Ferrucci JT Jr, Simeone JF, Butch RJ, Wittenberg J, White M, Brown AS: Lesser sac abscesses and fluid collections: drainage by transhepatic approach. Radiology 1985;155:615-618.

28 Gervais DA, Hahn PF, O'Neill MJ, Mueller PR $\mathrm{CT}$-guided transgluteal drainage of deep pelvic abscesses in children: selective use as an alternative to transrectal drainage. AJR Am J Roentgenol 2000; 175:1393-1396.

-29 Akinci D, Akhan O, Ozmen MN, Karabulut N, Ozkan O, Cil BE, Karcaaltincaba M: Percutaneous drainage of 300 intraperitoneal abscesses with long-term follow-up. Cardiovasc Intervent Radiol 2005;28:744-750.

30 Kim JK, Jeong YY, Kim YH, Kim YC, Kang HK, Choi HS: Postoperative pelvic lymphocele: treatment with simple percutaneous catheter drainage. Radiology 1999;212:390-394.

31 Butch RJ, Mueller PR, Ferrucci JT Jr, Wittenberg J, Simeone JF, White EM, Brown AS: Drainage of pelvic abscesses through the greater sciatic foramen. Radiology 1986;158:487-491. 
\$2 Maher MM, Gervais DA, Kalra MK, Lucey B, Sahani DV, Arellano R, Hahn PF, Mueller PR: The inaccessible or undrainable abscess: how to drain it. Radiographics 2004;24:717-735.

33 Michalson AE, Brown BP, Warnock NG, Simonson TM: Presacral abscesses: percutaneous transperineal drainage with use of bone landmarks and fluoroscopic guidance. Radiology 1994;190:574-575

-34 Sperling DC, Needleman L, Eschelman DJ, Hovseplan DM, Lev-Toaff AS: Deep pelvic abscesses: transperineal US-guided drainage. Radiology 1998; 208:111-115.

\35 Gazelle GS, Haaga JR, Stellato TA, Gauderer MW, Plecha DT: Pelvic abscesses: CT-guided transrectal drainage. Radiology 1991;181:49-51.

-36 Nosher JL, Needell GS, Amorosa JK, Krasna IH: Transrectal pelvic abscess drainage with sonographic guidance. AJR Am J Roentgenol 1986;146: 1047-1048.

37 Nielsen MB, Pedersen ST: Sonographically guided transrectal or transvaginal one-step catheter placement in deep pelvic and perirectal abscesses. AJR Am J Roentgenol 2004;183:1035-1036.

38 Bennett JD, Kozak RI, Taylor BM, Jory TA: Deep pelvic abscesses: transrectal drainage with radiologic guidance. Radiology 1992;185:825-828.

-39 Hovseplan DM, Steele JR, Skinner CS, Malden ES: Transrectal versus transvaginal abscess drainage: survey of patient tolerance and effect on activities of daily living. Radiology 1999;212:159-163.

40 Casola G, vanSonnenberg E, D'Agostino HB, Harker CP, Varney RR, Smith D: Percutaneous drainage of tuboovarian abscesses. Radiology 1992;182: 399-402.

41 Lang EK, Springer RM, Glorioso LW, Cammarata CA: Abdominal abscess drainage under radiologic guidance: causes of failure. Radiology 1986;159: 329-336.

42 Lieberman RP, Hahn FJ, Imray TJ, Phalen JT: Loculated abscesses: management by percutaneous fracture of septations. Radiology 1986;161:827-828.

-43 Johnson RD, Mueller PR, Ferrucci JT Jr, Dawson SL, Butch RJ, Papanicolaou N, vanSonnenberg E, Simeone JF, Wittenberg J: Percutaneous drainage of pyogenic liver abscesses. AJR Am J Roentgenol 1985;144:463-467.

44 Mueller PR, Ferrucci JT Jr, Wittenberg J, Simeone JF, Butch RJ: Iliopsoas abscess: treatment by CT-guided percutaneous catheter drainage. Am J Roentgenol 1984;142:359-362.
5 Steiner E, Mueller PR, Hahn PF, Saini S, Simeone JF, Wittenberg J, Warshaw AL, Ferrucci JT Jr: Complicated pancreatic abscesses: problems in interventional management. Radiology 1988;167: 443-446.

46 Tyrrel RT, Murphy FB, Bernardino ME: Tuboovarian abscesses: CT-guided percutaneous drainage. Radiology 1990;175:87-89.

47 vanSonnenberg E, Wittich GR, Casola G, Brannigan TC, Karnel F, Stabile BE, Varney RR, Christensen RR: Percutaneous drainage of infected and noninfected pancreatic pseudocysts: experience in 101 cases. Radiology 1998;170:757-761.

48 vanSonnenberg E, Wittich GR, Casola G, et al: Periappendiceal abscesses: percutaneous drainage. Radiology 1987;163:23-26.

49 Gervais DA, Brown SD, Connolly SA, Brec SL, Harisinghani MG, Mueller PR: Percutaneous imaging-guided abdominal and pelvic abscess drainage in children. Radiographics 2004;24:737-754.

50 Johannsen EC, Sifri CD, Madoff LC: Pyogenic liver abscess. Infect Clin North Am 2000;14:547-563.

51 Silver S, Weinstein A, Cooperman A: Changes in the pathogenesis and detection of intrahepatic abscess. Am J Surg 1979;137:608-610.

52 Chou FF, Sheen-Chen SM, Chen YS, Chen MC: Single and multiple pyogenic liver abscesses: clinical course, etiology, and results of treatment. World J Surg 1997;21:384-389.

53 Baek SY, Lee MG, Cho KS, Lee SC, Sung KB, Auh YH: Therapeutic percutaneous aspiration of hepatic abscesses: effectiveness in 25 patients. Am J Roentgenol 1993;160:799-802.

54 Greenberger NJ, Toskes PP, Isselbacher KJ: Diseases of the Pancreas. Harrison's Principles of Internal Medicine, ed 11. New York, McGraw-Hill, 1987, pp 1372-1384.

55 vanSonnenberg E, Wittich GR, Chon KS, D'Agostino HB, Easter D, Morgan RG, Walser EM, Nealon WH, Goodacre B, Stabile BE: Percutaneous radiologic drainage of panceatic abscesses. AJR Am J Roentgenol 1997;168:979-984.

56 Mithofer K, Mueller PK, Warshaw AL: Interventional and surgical treatment of pancreatic abscess. World J Surg 1997;21:162-168.

57 Barkin JS, Smith FR, Pereiras R Jr, Isikoff M, Levi J, Livingstone A, Hill M, Rogers AI: Therapeutic percutaneous aspiration of pancreatic pseudocysts. Dig Dis Sci 1981;26:585-586.
58 Grosso M, Gandini G, Cassinis MC, Regge D, Righi D, Rossi P: Percutaneous treatment (including pseudocystogastrostomy) of 74 panreatic pseudocysts. Radiology 1989;173:493-497.

59 Neff R: Pancreatic pseudocysts and fluid collections: percutaneous approaches. Surg Clin North Am 2001;81:399-403.

60 Mueller PR, Saini S, Wittenburg J, Simeone J, Hahn PF, Steiner E, Dawson SL, Butch BJ, Stark DD, Ottinger LW: Sigmoid diverticular abscesses: percutaneous drainage as an adjunct to surgical resection in 24 cases. Radiology 1987;164:321-325.

61 Butler JA, Huang J, Wilson SE: Repeated laparotomy for post-operative intra-abdominal sepsis: an analysis of outcome factors. Arch Surg 1987;122: 702-706.

62 Montgomery RS, Wilson SE: Intraabdominal abscess: image-guided diagnosis and therapy. Clin Infect Dis 1996;23:28-36.

63 Green BT: Splenic abscess: report of six cases and review of the literature. Am Surg 2001;67:80-84.

64 Phillips GS, Radosevich MD, Lipsett PA: Splenic abscess: another look at an old disease. Arch Surg 1997;132:1331-1336.

65 Hadas-Halpren I, Hiller N, Dolberg M: Percutaneous drainage of splenic abscesses: an effective and safe procedure. Br J Radiol 1992;65:968-970.

66 Schwerk WB, Gorg K, Restrepo I: Ultrasound-guided percutaneous drainage of pyogenic splenic abscesses. J Clin Ultrasound 1994;22:161-166.

67 Bouali K, Magotteaux P, Jadot A, Saive C, Lombard R, Weerts J, Dallemagne B, Jehaes C, Delforge M, Fontaine F: Percutaneous catheter drainage of abdominal abscess after abdominal surgery. Results in 121 cases. J Belg Radiol 1993;76: 11-14.

68 Wittich G: Radiological treatment of abdominal abscess with fistulous communications. Curr Opin Radiol 1992;4:110-115.

69 Gerzof SG, Johnson WC, Robbins AH, Nasbeth DC: Expanded criteria for percutaneous abscess drainage. Arch Surg 1985;120:227-232.

70 Marano I, Mainenti PP, Selva G, Cannavale M, Sodano A: Computerized tomography-guided drainage of postoperative abdominal fluid collection. Radiol 1999;97:160-165. 